Herman Veenhof, Remco A. Koster, Jan-Willem C. Alffenaar, Aad P. van den Berg, Marco R. de Groot, Erik A.M. Verschuuren, Stefan P. Berger, Stephan J.L. Bakker and Daan J. Touw*

\title{
Clinical application of a dried blood spot assay for sirolimus and everolimus in transplant patients
}

https://doi.org/10.1515/cclm-2019-0053

Received January 15, 2019; accepted March 8, 2019; previously published online April 2, 2019

\section{Abstract}

Background: Monitoring of immunosuppressive drugs such as everolimus and sirolimus is important in allograft rejection prevention in transplant patients. Dried blood spots (DBS) sampling gives patients the opportunity to sample a drop of blood from a fingerprick at home, which can be sent to the laboratory by mail.

Methods: A total of 39 sirolimus and 44 everolimus paired fingerprick DBS and whole blood (WB) samples were obtained from 60 adult transplant patients for method comparison using Passing-Bablok regression. Bias was assessed using Bland-Altman. Two validation limits were pre-defined: limits of analytical acceptance were set at $>67 \%$ of all paired samples within $20 \%$ of the mean of both samples and limits of clinical relevance were set in

*Corresponding author: Daan J. Touw, PharmD, PhD, Department of Clinical Pharmacy and Pharmacology, University of Groningen, University Medical Center Groningen, PO Box 30.001, 9700 RB Groningen, The Netherlands; and Department of Pharmacy, Section Pharmacokinetics, Toxicology and Targeting, University of Groningen, Groningen, The Netherlands, Phone: +31503614071, Fax: +31 503612417, E-mail: d.j.touw@umcg.nl

Herman Veenhof and Jan-Willem C. Alffenaar: Department of Clinical Pharmacy and Pharmacology, University of Groningen, University Medical Center Groningen, Groningen, The Netherlands

Remco A. Koster: Department of Clinical Pharmacy and Pharmacology, University of Groningen, University Medical Center Groningen, Groningen, The Netherlands; and PRA Health Sciences, Bioanalytical Laboratory, Assen, The Netherlands

Aad P. van den Berg: Department of Gastroenterology and Hepatology, University of Groningen, University Medical Center Groningen, Groningen, The Netherlands

Marco R. de Groot: Department of Hematology, University of Groningen, University Medical Center Groningen, Groningen, The Netherlands

Erik A.M. Verschuuren: Department of Pulmonology and Tuberculosis, University of Groningen, University Medical Center Groningen, Groningen, The Netherlands

Stefan P. Berger and Stephan J.L. Bakker: Department of Internal Medicine, Division of Nephrology, University of Groningen, University Medical Center Groningen, Groningen, The Netherlands a multidisciplinary team at $>80 \%$ of all paired samples within $15 \%$ of the mean of both samples.

Results: For both sirolimus and everolimus, PassingBablok regression showed no differences between WB and DBS with slopes of 0.86 (95\% CI slope, 0.72-1.02) and 0.96 (95\% CI 0.84-1.06), respectively. Only everolimus showed a significant constant bias of $4 \%$. For both sirolimus and everolimus, limits of analytical acceptance were met $(76.9 \%$ and $81.8 \%$, respectively), but limits or clinical relevance were not met ( $77.3 \%$ and $61.5 \%$, respectively).

Conclusions: Because pre-defined limits of clinical relevance were not met, this DBS sampling method for sirolimus and everolimus cannot replace WB sampling in our center at this time. However, if the clinical setting is compatible with less strict limits for clinical relevance, this DBS method is suitable for clinical application.

Keywords: dried blood spots; immunosuppressants; microsampling; validation.

\section{Introduction}

Lifelong therapy with immunosuppressive drugs is a cornerstone in the prevention of rejection of allografts in transplant patient care [1]. Because of their narrow therapeutic range, many immunosuppressive drugs, including the mammalian target of rapamycin inhibitors everolimus and sirolimus are subject to therapeutic drug monitoring (TDM) to allow for balancing between toxic- and subtherapeutic drug concentrations. Tacrolimus is currently the most widely used calcineurin inhibitor in kidney transplant patient care [2]. However, the recent TRANSFORM trial suggests efficacy of maintenance therapy with everolimus in combination with low dose tacrolimus is comparable to a standard regimen of tacrolimus and mycophenolate mofetil [3, 4]. An additional advantage is the reduced viral infection risk. This might lead to an increase in everolimus use in transplant patients.

Traditionally, venous blood samples are used for monitoring of immunosuppressive drug concentrations and patients have to travel to the hospital on a regular basis to have their blood drawn. To decrease the burden 
for patients, dried blood spot (DBS) home sampling has been developed among various micro sampling methods for several drugs, including immunosuppressants, to enable home sampling [5-16]. For this, a drop of blood from a fingerprick is applied to a sampling card and dried. This card is sent to the laboratory by mail a few days prior to routine check-up of the patient in the hospital. At the time of the check-up, blood-drug concentrations and creatinine levels will be available for the clinician and the patient. Current challenges of DBS implementation include the influence of the hematocrit and logistical hurdles $[9,13,17,18]$. Although DBS analytical methods can meet the required analytical standards, analysis of clinically collected samples does not always result in sufficient agreement between the standard (venous) method and the novel fingerprick DBS method [17]. Therefore, a clinical validation study showing interchangeability between DBS and venous sampling is required before clinical application [18]. This is shown for tacrolimus, cyclosporin A and creatinine [5, 7-15, 19]. For sirolimus, Dickerson et al. report agreement between fingerprick DBS and venous samples in 25 pediatric transplant patients, where mean DBS concentrations were on average $0.8 \mu \mathrm{g} / \mathrm{L}$ lower than venous samples [15]. This difference between the two methods increased with increasing concentrations of sirolimus. Willemsen et al. reported agreement between everolimus fingerprick DBS and venous samples in 20 patients with cancer with a mean ratio of whole blood (WB) to DBS concentrations of 0.90 [20]. The current Clinical and Laboratory Standards Institute (CLSI) guideline suggests at least 40 paired samples for comparison, therefore, the number of samples collected in both studies for cross-validation was low [21]. In addition, no clinical validation study for everolimus using fingerprick DBS has been published for transplant patients. Therefore, the aim of this study was to clinically validate our method for analyzing sirolimus and everolimus in DBS to enable implementation in routine care.

\section{Materials and methods}

\section{Patients and sample collection}

Patient samples were collected from adult transplant patients during routine clinical check-ups in the hospital. Because of the nature of this study, the need to provide written informed consent by the patients was waived by the Ethics Committee of the University Medical Center Groningen (Metc 2011.394). A trained phlebotomist obtained both the venous and DBS samples within $10 \mathrm{~min}$ of each other using a collection method described elsewhere [9, 22, 23]. In short, after a fingerprick, two drops of blood were allowed to fall freely on a Whatman FTA DMPK-C sampling paper (GE Healthcare, Chicago, IL, USA). The WB samples were analyzed within a day as they were part of routine care. DBS are stable for at least 7 days at room temperature, therefore the DBS samples were allowed to dry for 24-74 $\mathrm{h}$ at room temperature and packed in zip lock plastic mini bags with a desiccant [24-26]. Upon receiving the DBS samples in the laboratory, the samples were inspected for spot quality based on predefined criteria [22, 23, 27]. DBS samples fit for analysis were stored at $-20^{\circ} \mathrm{C}$ until analysis. DBS samples are stable for at least 29 weeks at $-20^{\circ} \mathrm{C}$ so analysis occurred within this timeframe [25].

\section{Equipment, conditions and procedures}

Our reference procedure was a measurement of sirolimus and everolimus in WB obtained by venipuncture, with a previously validated analysis method performed on a Thermo Fisher Scientific (Waltham, MA, USA) triple quadrupole Quantiva LC-MS/MS system with a Vanquish HPLC system [28]. For the DBS analysis of sirolimus and everolimus, a previously validated method was used using the aforementioned Thermo Quantiva LC-MS/MS [24, 25]. The analytical range for both the WB and DBS assay for sirolimus and everolimus was $1.0-50.0 \mu \mathrm{g} / \mathrm{L}$.

Hematocrit of the venous samples was measured using an XN10/XN20 hematology analyzer (Sysmex, Kobe, Japan).

\section{Statistical analysis}

Statistical analysis was performed using Analyse-it ${ }^{\circledR}$ Method Validation Edition for Microsoft Excel version 4.18.6 (Analyse-it, Leeds, UK) and Microsoft Excel (Microsoft Inc., Redmond, WA, USA). Method comparison was done using Passing-Bablok regression analysis and a Bland-Altman analysis was used for bias calculation [29, 30]. Because no official guideline exists for clinical validation of DBS assays, we set two limits of acceptance $a$ priori. The first is the limit of analytical acceptance which is based on the EMA guidelines for cross-validation and the 2018 version of the FDA guideline for studies required to bridge two analytical methods [31, 32]. As acceptance criteria, both FDA and EMA guidelines state that at least two-thirds (67\%) of the paired samples should be $\pm 20 \%$ of the mean of both methods. The second is the limit of clinical relevance which was set at a range of $85 \%-115 \%$ around the ratio of the paired DBS and WB samples for at least $80 \%$ of the samples. This range was chosen by a multidisciplinary team consisting of clinicians, pharmacists and analysts and was chosen based on the therapeutic window given in the summary of product characteristics of 3-8 $\mu \mathrm{g} / \mathrm{L}$ for everolimus and 4-12 $\mu \mathrm{g} / \mathrm{L}$ for sirolimus trough concentrations for stable transplant patients $>3$ months after transplantation [33, 34]. A difference of $15 \%$ in the acceptable range ratio for a high everolimus trough concentration $(8 \mu \mathrm{g} / \mathrm{L})$ in WB would lead to a DBS concentration range of $6.8-9.2 \mu \mathrm{g} / \mathrm{L}$. For a low everolimus trough concentration $(3 \mu \mathrm{g} / \mathrm{L})$ in WB this would lead to an acceptable DBS concentration range of $2.6-3.5 \mu \mathrm{g} / \mathrm{L}$. These values are comparable to the acceptable variability of $15 \%$ for accuracy and precision that are mentioned in the FDA and EMA guidelines for bioanalytical methods [31, 32]. If $80 \%$ of all patients are within this range this was deemed feasible by the clinicians. 
The predictive performance of the DBS analytical method was established using the method described by Sheiner and Beal [35]. In short, DBS concentrations were used to predict WB concentrations. For each paired WB and DBS sirolimus and everolimus sample, the slope and intercept of the Passing-Bablok regression was calculated using the whole population of sirolimus and everolimus samples, respectively, excluding the data of that specific paired sample. The error of this prediction is determined by bias and imprecision. The bias is the median difference between the predicted and true concentration and is shown by the median prediction error (MPE) and the median percentage prediction error (MPPE). The imprecision is the variance of the predicted values which is measured by the root median squared prediction error (RMSE) and the median absolute percentage prediction error (MAPE). For analyzing the predictive performance the following equations were used:

median prediction error $(\mathrm{MPE})=$ median $($ predicted $\mathrm{WB}-\mathrm{WB})$

median percentage prediction error $(\mathrm{MPPE})=$

$$
\text { median }\left(100 \% * \frac{\text { predicted WB-WB }}{\text { WB }}\right)
$$

root median squared prediction error $(\mathrm{RMSE})=$

$$
\sqrt{\text { median }}(\text { predicted WB }-\mathrm{WB})^{2}
$$

median absolute percentage prediction error $(\mathrm{MAPE})=$

$$
\text { median }\left(100 \% * \frac{\mid \text { predicted WB }-\mathrm{WB} \mid}{\mathrm{WB}}\right)
$$

In accordance with other studies, acceptable values for MPPE and MAPE were set at $<15 \%$ and at least $67 \%$ of all samples should have an absolute prediction error of $<20 \%[5,20]$.

\section{Results}

\section{Patients and samples}

A total of 90 paired DBS and WB samples were taken from 60 adult transplant patients between January 2017 and December 2017. All DBS cards had at least one spot of sufficient quality for analysis. Three samples were excluded because no paired WB sample was taken. Another three samples were excluded because the WB and DBS sample were not taken within $10 \mathrm{~min}$ of each other. One sample was excluded because it was not a trough concentration. A total of 39 paired sirolimus and 44 paired everolimus samples were available for method comparison from 29 and 27 unique transplant patients, respectively. The hematocrit ranged from 0.23 to $0.51(\mathrm{v} / \mathrm{v})$ with a mean hematocrit of 0.40 . All hematocrit values were within the analytically validated range, which means that the hematocrit value had no influence on the DBS analytical results [24]. Mean concentrations of sirolimus and everolimus in WB and DBS can be found in Table 1. All evaluated concentrations
Table 1: Mean drug concentrations, range and SD of sirolimus and everolimus in WB and DBS.

\begin{tabular}{lcr}
\hline Drug concentrations & $\mathbf{n}$ & Mean \pm SD (range) \\
\hline Sirolimus in WB, $\mu \mathrm{g} / \mathrm{L}$ & 39 & $5.0 \pm 2.4(1.9-10.9)$ \\
Sirolimus in DBS, $\mu \mathrm{g} / \mathrm{L}$ & 39 & $4.7 \pm 1.9(1.8-9.7)$ \\
Everolimus in WB, $\mu \mathrm{g} / \mathrm{L}$ & 44 & $5.4 \pm 2.6(1.2-14.3)$ \\
Everolimus in DBS, $\mu \mathrm{g} / \mathrm{L}$ & 44 & $5.0 \pm 2.4(1.9-10.9)$ \\
\hline
\end{tabular}

DBS, dried blood spot; SD, standard deviation; WB, whole blood.

Table 2: Patient demographics and transplantation type.

\begin{tabular}{lcl}
\hline $\begin{array}{l}\text { Patient demographics and } \\
\text { clinical laboratory data }\end{array}$ & $\mathbf{n}$ & Median (range) \\
\hline $\begin{array}{l}\text { Age, years } \\
\text { Sex }\end{array}$ & 56 & $\begin{array}{l}61(23-77) \\
38 \text { male }(67.9 \%), \\
18 \text { female }(32.1 \%)\end{array}$ \\
Time from transplantation & 56 & $\begin{array}{l}\text { 2 years, 3 months, 5 days } \\
\text { (10 days-22 years, 7 months) }\end{array}$ \\
\hline
\end{tabular}

Table 3: Patient transplantation type per sample type.

\begin{tabular}{lrrrr}
\hline $\begin{array}{l}\text { Transplantation } \\
\text { type }\end{array}$ & $\begin{array}{r}\text { Sirolimus } \\
\text { samples }\end{array}$ & $\begin{array}{r}\text { Everolimus } \\
\text { samples }\end{array}$ & $\begin{array}{r}\text { Total } \\
\text { samples }\end{array}$ & $\begin{array}{r}\text { Unique } \\
\text { patients }\end{array}$ \\
\hline Liver & 30 & 0 & 30 & 22 \\
Lung & 2 & 7 & 9 & 7 \\
Stem cell & 7 & 0 & 7 & 6 \\
Kidney & 0 & 37 & 37 & 21 \\
Total & 39 & 44 & 83 & 56 \\
\hline
\end{tabular}

were within the analytically validated range [24]. Patient demographics and transplantation type can be found in Tables 2 and 3.

\section{Clinical validation}

\section{Sirolimus}

For sirolimus, the Passing-Bablok analysis fit was $y=0.86 x+0.44$ (95\% CI slope, 0.72-1.02; 95\% CI intercept -0.23 to 1.11 ) showing no significant constant or systematic difference as can be seen in Figure 1. The correlation coefficient was 0.93. The Bland-Altman plot (Figure 2) shows that the mean ratio of WB and DBS sirolimus concentrations is 1.00 (95\% CI 0.93-1.07), without significant bias. The $95 \%$ limits of agreement (LoA) are 0.60 and 1.40 , which is wider than the limits of analytical acceptance which were set at 0.80 and 1.20. Nine out of 39 values 


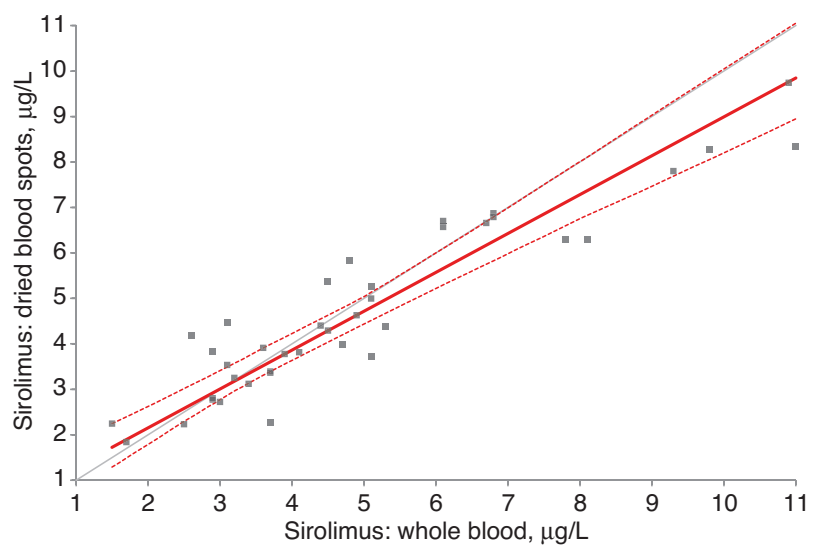

Figure 1: Method comparison for sirolimus concentrations in WB and DBS $(n=39)$.

The continuous line is the Passing-Bablok regression line $\mathrm{y}=0.86 \mathrm{x}+0.44(95 \% \mathrm{Cl}$ slope, $0.72-1.02 ; 95 \% \mathrm{Cl}$ intercept -0.23 to 1.11). The dashed line is the $95 \% \mathrm{Cl}$.
(23.1\%) fell outside the limits of analytical acceptance. For the limits of clinical relevance this was 15/39 (38.5\%). For the predictive performance, bias was small with an MPE of $-0.008 \mu \mathrm{g} / \mathrm{L}$ and an MPPE of $-0.16 \%$. The predictive performance of imprecision as measured by the RMSE was small with a value of $0.56 \mu \mathrm{g} / \mathrm{L}$. The MAPE was within acceptable limits $(<15 \%)$ with a value of $11.07 \%$. The acceptance limit for MAPE (at least $67 \%$ of the samples with a value $<20 \%$ ) was met with 30 out of 39 values (76.9\%) (Figure 3).

\section{Everolimus}

For everolimus, the Passing-Bablok analysis fit was $\mathrm{y}=0.96 \mathrm{x}+0.37$ (95\% CI slope, 0.84-1.06; 95\% CI intercept -0.11 to 0.99 ), also showing no significant constant or systematic difference as can be seen in Figure 4.

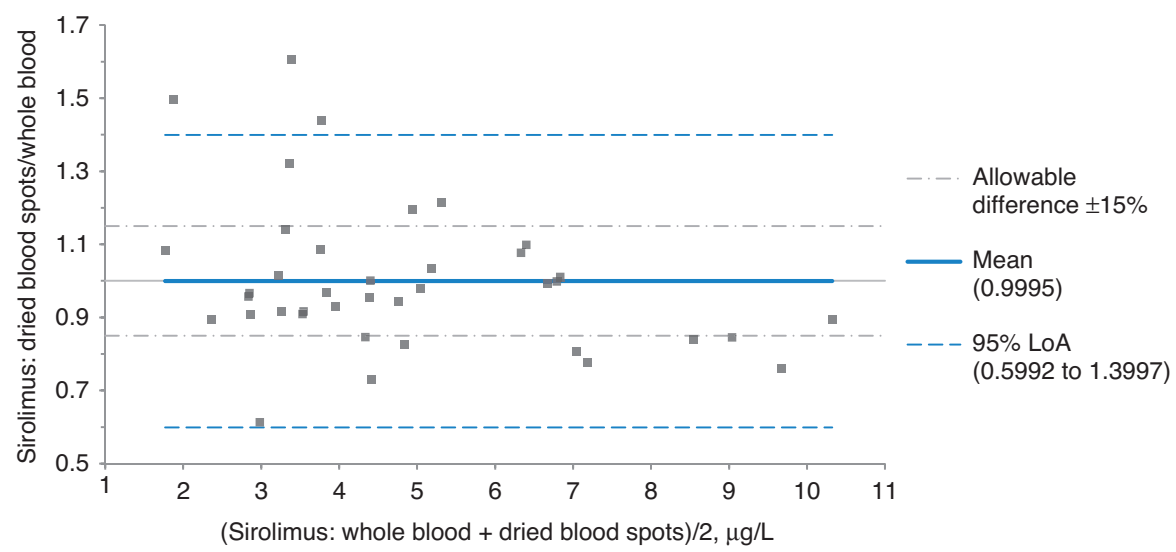

Figure 2: Method comparison for sirolimus concentrations in WB and DBS $(n=39)$.

The continuous line is the Bland-Altman bias estimation of 1.00 ( $95 \% \mathrm{Cl} 0.93-1.07)$. The dashed line is the $95 \%$ LoA and the dotted/dashed line is the limit of clinical relevance set at $15 \%$.

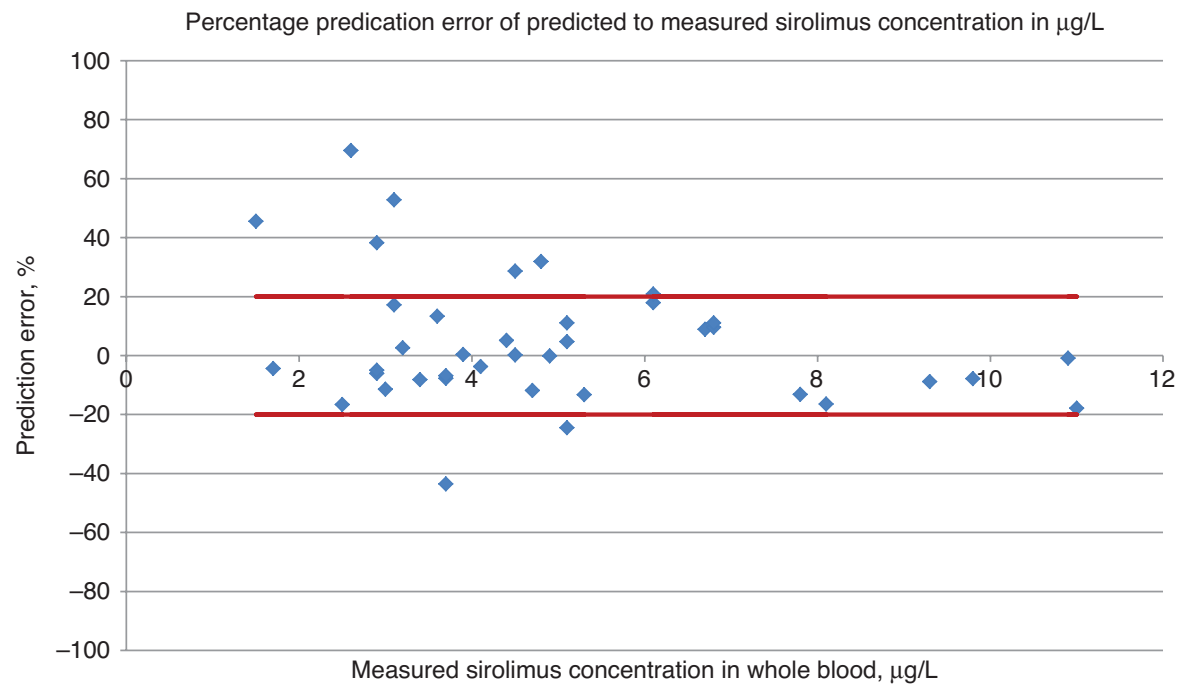

Figure 3: Percentage prediction error or predicted to measured sirolimus concentrations with acceptable prediction error set at $-20 \%$ and $20 \%$. 


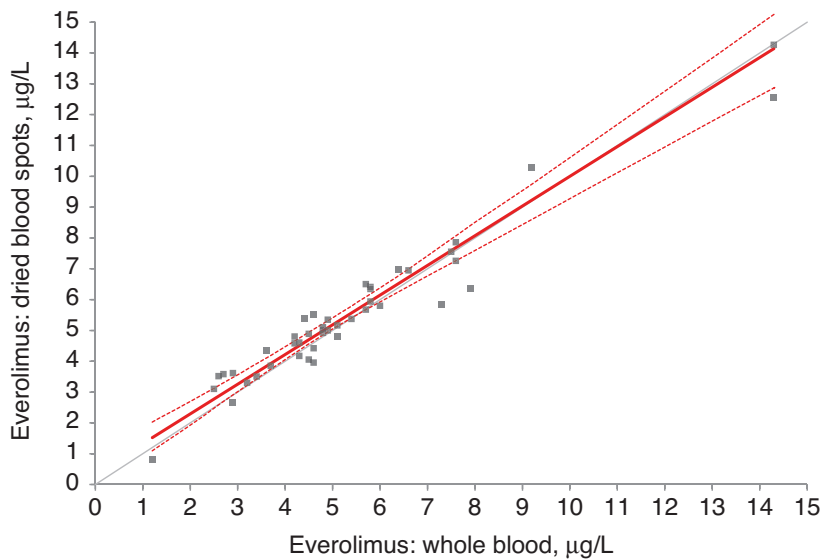

Figure 4: Method comparison for everolimus concentrations in WB and DBS $(n=44)$.

The continuous line is the Passing-Bablok regression line $\mathrm{y}=0.96 \mathrm{x}+0.37$ ( $95 \% \mathrm{Cl}$ slope, $0.84-1.06 ; 95 \% \mathrm{Cl}$ intercept -0.11 to 0.99). The dashed line is the $95 \% \mathrm{Cl}$.

The correlation coefficient was 0.97. The Bland-Altman plot (Figure 5) shows that the mean ratio of WB and DBS everolimus concentrations is 1.04 (95\% CI 1.00-1.08), which is a small but significant bias of $4 \%$. The $95 \%$ LoA are 0.78 and 1.30 , which is wider than the limits of analytical acceptance which were set at 0.80 and 1.20 . Only eight out of 44 values (18.2\%) fell outside the limits of analytical acceptance. For the limits of clinical relevance this was 10 out of $44(22.7 \%)$. For the predictive performance, bias was small with an MPE of $0.003 \mu \mathrm{g} / \mathrm{L}$ and an MPPE of $0.13 \%$. The imprecision as measured by the RMSE was small with a value of $0.39 \mu \mathrm{g} / \mathrm{L}$. The MAPE was within acceptable limits $(<15 \%)$ with a value of $7.9 \%$. The acceptance limit for MAPE (at least $67 \%$ of the samples with a value $<20 \%$ ) was met with 39 out of 44 values (88.6\%) (Figure 6). One

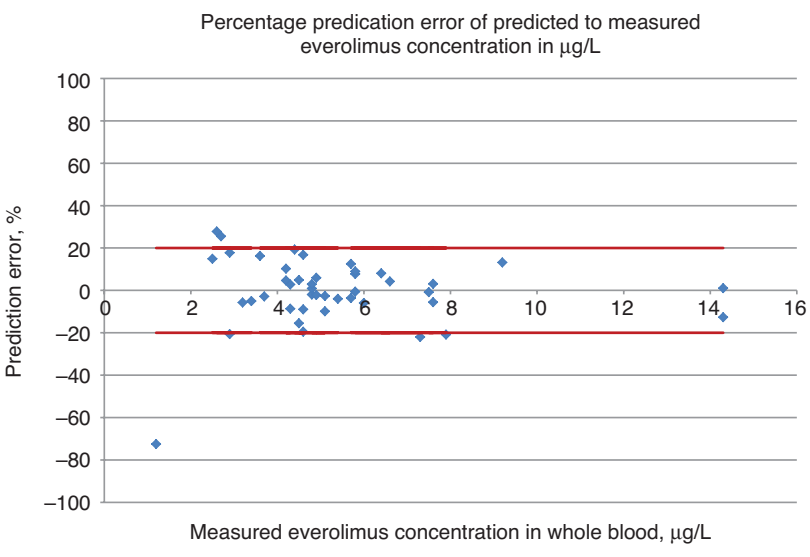

Figure 6: Percentage prediction error or predicted to measured everolimus concentrations with acceptable prediction error set at $-20 \%$ and $20 \%$.

outlier of $-72.5 \%$ was observed. The outlier prediction error shown in Figure 6 can likely be explained by the low concentration of everolimus (1.2 $\mathrm{gg} / \mathrm{L}$ in WB), which is just above the lower limit of quantification of the method. In this setting, the influence of the intercept $(-0.49)$ becomes paramount, resulting in a predicted value of $0.33 \mu \mathrm{g} / \mathrm{L}$, giving a prediction error of $-72.5 \%$.

\section{Discussion}

This study showed good agreement between DBS sirolimus and everolimus concentrations and venous WB concentrations in transplant patients over a concentration range relevant for TDM of trough concentrations. No correction factor is needed to calculate WB values from DBS

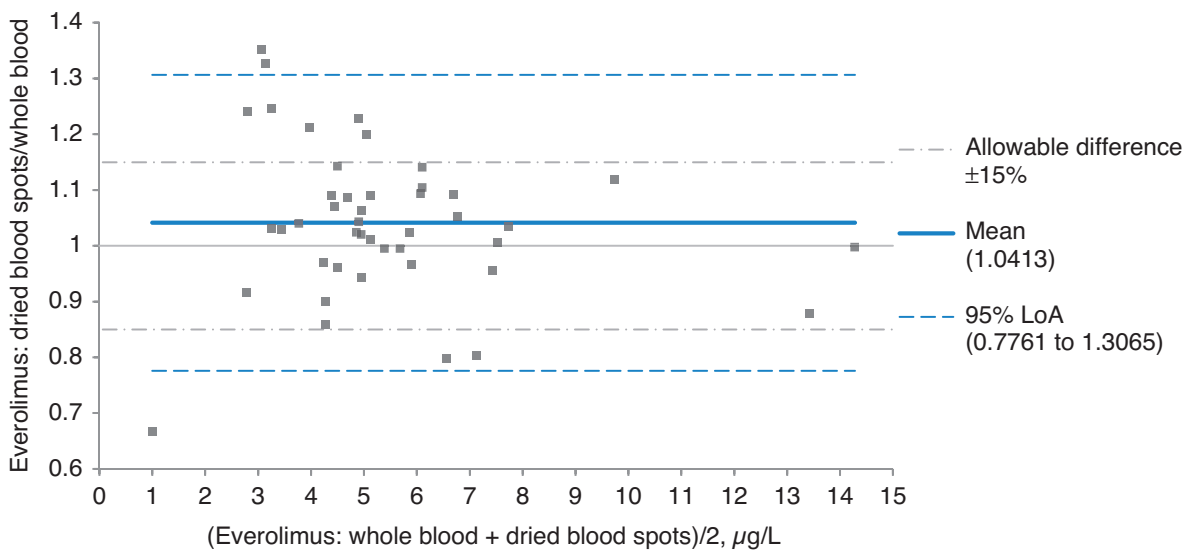

Figure 5: Method comparison for everolimus concentrations in WB and DBS $(n=44)$. The continuous line is the Bland-Altman bias estimation of $1.05(95 \% \mathrm{Cl} 1.00-1.08)$. The dashed line is the $95 \%$ LoA and the dotted/dashed line is the limit of clinical relevance set at $15 \%$. 
values. For sirolimus and everolimus $76.9 \%$ and $81.8 \%$, respectively, of all DBS concentrations fell within limits of analytical acceptance. Therefore, this method met the requirements set in the EMA guideline for cross-validation and FDA guidelines for bridging studies [31, 32]. The predictive performance of the sirolimus and everolimus DBS method complied with the predefined criteria of $>67 \%$ of all samples to have a prediction error of $<20 \%$. However, the limits set for clinical relevance ( $>80 \%$ of the samples with $<15 \%$ of the mean) were not met with a value of $77.3 \%$ and $61.5 \%$ for sirolimus and everolimus, respectively.

Because tacrolimus is the most widely used immunosuppressant in our center to prevent renal allograft rejection, the amount of patients in our institution receiving either sirolimus or everolimus is limited. Therefore, patients from all transplantation types (Table 3) were asked to provide samples. The heterogeneous patient population is a strength of this study, hematocrit values of all patients were within the analytically validated limits and mean hematocrit values were comparable between the different groups of transplant patients (data not shown).

Because a clinical validation of a DBS fingerprick method shows strong resemblance to a cross validation, the CLSI guideline recommends to include at least 40 patient samples [21]. Although the study by Willemsen et al. showed good agreement between WB and capillary blood, the performed power calculation resulting in 20 samples necessary was done prior to this result. The power calculation was based on the assumption that venous blood and DBS are the same matrix and no effect of the hematocrit is expected [20]. It is, however, well-known that hematocrit can affect DBS assays and sometimes results in unacceptable biases [24, 25, 36]. Capillary collected blood consists of a mixture of venous blood, arterial blood and interstitial fluid which is not the same matrix as a venous WB sample. Therefore, we think making an assumption that the matrix of capillary blood is the same as venous WB is not recommended. Following the CLSI guideline for finding a sample size would, in our opinion, be more appropriate. The recommendation of 40 samples in the CLSI guideline is based on regression analysis described by Linnet, where the amount of samples necessary for a cross-validation can be calculated based on the analytical characteristics of the assay [37]. If Linnets' calculation would be followed for the everolimus DBS assay used by Willemsen et al., the recommended number of samples is 40 , and if Linnets' calculation would be followed for the sirolimus DBS assay used by Dickerson et al. the recommended number of samples is $37[19,36]$. Because of the exclusion of several sirolimus samples the required amount of 40 samples was not met. However, with the amount of 39 paired samples available, we do not think that the absence of one paired sample has a great influence on the clinical validation.

For everolimus, our results are in part in agreement with Willemsen et al. [20]. Our method did not show a constant or proportional bias as shown by Willemsen et al. where a small but significant proportional bias was found in the Passing-Bablok regression. In addition, they demonstrated a ratio of 0.90 in the Bland-Altman comparison, where our method shows a small but statistically significant ratio of 1.04. It should be noted that the BlandAltman comparison by Willemsen et al. is shown as a ratio of WB/DBS which is in contrast with this study where the ratio is shown as DBS/WB. However, the spread of the relative difference in our method (Figure 5) and corresponding LoAs are wider than in the method used by Willemsen et al. This is especially true for the low trough concentrations $(1-5 \mu \mathrm{g} / \mathrm{L})$. Although not statistically significant, the analytical validation showed a trend towards more bias at lower concentrations ( $3 \mu \mathrm{g} / \mathrm{L})$ compared to higher concentrations $(10 \mu \mathrm{g} / \mathrm{L})$ for everolimus [24]. This might be an explanation for the observed spread of relative difference. Other clinical validation studies usually have few samples and very few samples in the low concentrations range. However, in a study on tacrolimus, $22.2 \%(n=63)$ of the lower (trough) concentrations exceeded $\pm 20 \%$ limits of the DBS to WB concentration ratio [5]. In this study, the area under the curve (AUC) was calculated for both DBS and WB based on trough concentrations and three sampling points at $\mathrm{t}=1, \mathrm{t}=2$ and $\mathrm{t}=3 \mathrm{~h}$ after medication intake. For the AUCs, $90.3 \%(n=63)$ of the paired AUC values were within 20\% limits of DBS to WB ratio suggesting higher tacrolimus concentrations show less spread compared to trough concentrations. It is unlikely that the hematocrit has caused these differences, because previous research shows that hematocrit effects are most prominent at high concentrations of everolimus and sirolimus $(50 \mu \mathrm{g} / \mathrm{L})$ and low hematocrits $(<0.23 \mathrm{v} / \mathrm{v})[24,25]$. Re-evaluation of the data stratified for either transplantation type or time from transplantations showed that these two factors are not of influence on the results (data not shown). In future studies, introduction of duplicate analysis of both WB and DBS samples or analysis of two individual blood spots might reduce the observed spread in the lower $(1-5 \mu \mathrm{g} / \mathrm{L})$ concentration range. In addition, incurred sample reanalysis (ISR) is recommended for both WB and DBS samples to assess the spread of individual patient samples. Two major differences present in the study by Willemsen et al. are the much broader concentration range of trough concentration samples (3.6-28.5 $\mu \mathrm{g} / \mathrm{L}$ in WB) and the broader 
limits of clinical relevance that were used in comparison to this study [20]. Because dosing of everolimus in patients with cancer is performed in steps of $2.5 \mathrm{mg}$ and the target trough concentration range is much wider (up to $19.2 \mu \mathrm{g} / \mathrm{L}$ ), a larger clinical limit is accepted [38, 39]. In transplant patients, dosing can be done in steps of $0.25 \mathrm{mg}$ and the target trough concentration range is $3-8 \mu \mathrm{g} / \mathrm{L}$, therefore, a much narrower limit of clinical relevance is adjudicated. To the best of our knowledge, no guideline is available to determine limits of clinical relevance for DBS. The available literature suggests that setting a limit of clinical relevance should be done in a multidisciplinary team taking into account the clinical application of the method, the patient characteristics and the properties of the analytical methods $[5,20]$. In our study, the everolimus DBS method does not meet the limits of clinical relevance set by our team and, at this time, cannot replace conventional WB sampling in the TDM of transplant patients where low trough concentrations are targeted. For sirolimus, Dickerson et al. showed a statistically significant difference of $-0.8 \mu \mathrm{g} / \mathrm{L}$ in the Bland-Altman analysis where our method showed no bias [15]. The range of sirolimus concentrations in Dickerson et al. is $4-18 \mu \mathrm{g} / \mathrm{L}$ which is higher than the range of 1.7-10.9 $\mu \mathrm{g} / \mathrm{L}$ in our study. The observed increased bias for higher trough concentrations ( $>10 \mu \mathrm{g} / \mathrm{L}$ ) shown in Dickerson et al. might also be present using our method. Although results are shown as a ratio, samples with a WB concentration of $>7.5 \mu \mathrm{g} / \mathrm{L}(\mathrm{n}=6)$ also showed lower concentrations in DBS (Figure 1) in this study. Excluding these samples yields a slope of 1.04 in Passing-Bablok regression, this explains the observed slope of 0.86 in the Passing-Bablok regression analysis for all sirolimus samples. However, excluding these samples does still result in not meeting the limits of clinical relevance. Another possibility is that this is a random phenomenon because the amount of samples with sirolimus WB concentration $>7.5 \mu \mathrm{g} / \mathrm{L}$ is limited. Additional samples in the range of $5-15 \mu \mathrm{g} / \mathrm{L}$ are needed to assess this. For sirolimus, the limits of clinical relevance are not met in this study and the same trend as for everolimus is present where samples with a concentration of $1-5 \mu \mathrm{g} / \mathrm{L}$ showed the greatest bias. This might be caused by the same factors mentioned before for everolimus. Therefore, at this time, the sirolimus DBS method cannot replace conventional WB sampling in the TDM of transplant patients with low trough concentrations.

In our study the DBS samples were obtained by trained phlebotomists at the hospital and not by the patients themselves at home. Considering DBS methods are intended for home-sampling this might be a limitation of our study. However, the instructions and sampling methods are the same for both phlebotomist and patient. Patients receive instructions before home sampling is initiated including practicing a fingerprick under the supervision of a trained phlebotomist. This should be sufficient for appropriate sampling at home if a patient or caregiver is willing and able to perform home sampling, in addition, paper and video instruction are available [40].

In the area of transplantation, where narrow therapeutic windows are followed for TDM of immunosuppressants, there are strict requirements for the analytical performance of assays measuring immunosuppressants in blood. With the current data, this clinical DBS validation study showed that not all predefined requirements set were met. Although Passing-Bablok analysis showed no systematic or constant differences between WB and DBS samples, the spread of samples did not meet the predefined limits of clinical relevance. However, as these limits were set by a local multidisciplinary team these may vary between settings and centers [18]. In addition, in a limited resources setting, where no WB bioanalytical method exists for sirolimus and everolimus, the DBS assay presented here could be used to allow TDM. If future studies show optimization of DBS assays using ISR, and if logistical challenges surrounding DBS home sampling can be overcome, the DBS method could be implemented in routine transplant patient care $[9,13,18]$. This would help in reducing patient burden, quickly achieving target trough levels the first months after transplantation and flexible monitoring of graft function.

Acknowledgment: We would like to thank the phlebotomists of the 'prikpoli' of the UMCG for obtaining the DBS samples.

Author contributions: All the authors have accepted responsibility for the entire content of this submitted manuscript and approved submission.

Research funding: This work was supported by the Netherlands Organization for Health Research and Development (ZonMw, The Hague, The Netherlands) Funder id: http:// dx.doi.org/10.13039/501100001826, grant 836044004 and the Tekke Huizinga Fund. This study was performed using the infrastructure and data provided by the TransplantLines Biobank and Cohort Study, which is registered at ClinicalTrials.gov under Identifier NCT03272841.

Employment or leadership: None declared.

Honorarium: None declared.

Competing interests: The funding organization(s) played no role in the study design; in the collection, analysis, and interpretation of data; in the writing of the report; or in the decision to submit the report for publication. 


\section{References}

1. Opelz G, Döhler B. Effect on kidney graft survival of reducing or discontinuing maintenance immunosuppression after the first year posttransplant. Transplantation 2008;86:371-6.

2. Kidney Disease: Improving Global Outcomes (KDIGO) Transplant Work Group. KDIGO clinical practice guideline for the care of kidney transplant recipients. Am J Transplant 2009;9(Suppl 3): S1-155.

3. Pascual J, Srinivas TR, Chadban S, Citterio F, Oppenheimer F, Tedesco H, et al. TRANSFORM: a novel study design to evaluate the effect of everolimus on long-term outcomes after kidney transplantation. J Clin Trials 2014;6:45-54.

4. Pascual J, Berger SP, Witzke O, Tedesco H, Mulgaonkar S, Qazi Y, et al. Everolimus with reduced calcineurin inhibitor exposure in renal transplantation. J Am Soc Nephrol 2018;29:1979-91.

5. Zwart TC, Gokoel SR, van der Boog PJ, de Fijter JW, Kweekel DM, Swen JJ, et al. Therapeutic drug monitoring of tacrolimus and mycophenolic acid in outpatient renal transplant recipients using a volumetric dried blood spot sampling device. Br J Clin Pharmacol 2018;84:2889-902.

6. Verheijen R, Thijssen B, Atrafi F, Schellens J, Rosing H, de Vries N, et al. Validation and clinical application of an LC-MS/MS method for the quantification of everolimus using volumetric absorptive microsampling. J Chromatography B 2019;1104:234-9.

7. Webb NJ, Roberts D, Preziosi R, Keevil BG. Fingerprick blood samples can be used to accurately measure tacrolimus levels by tandem mass spectrometry. Pediatr Transplant 2005;9:729-33.

8. van Boekel GA, Donders AR, Hoogtanders KE, Havenith TR, Hilbrands LB, Aarnoutse RE. Limited sampling strategy for prolonged-release tacrolimus in renal transplant patients by use of the dried blood spot technique. Eur J Clin Pharmacol 2015;71:811-6.

9. Veenhof H, Koster RA, Alffenaar JW, Berger SP, Bakker SJ, Touw DJ. Clinical validation of simultaneous analysis of tacrolimus, cyclosporine $A$ and creatinine in dried blood spots in kidney transplant patients. Transplantation 2017;101:1727.

10. Martial LC, Hoogtanders KE, Schreuder MF, Cornelissen EA, van der Heijden J, Joore MA, et al. Dried blood spot sampling for tacrolimus and mycophenolic acid in children: analytical and clinical validation. Ther Drug Monit 2017;39:412-21.

11. Leino AD, King EC, Jiang W, Vinks AA, Klawitter J, Christians U, et al. Assessment of tacrolimus intra-patient variability in stable adherent transplant recipients: establishing baseline values. Am J Transplant 2018.

12. Koop DR, Bleyle LA, Munar M, Cherala G, Al-Uzri A. Analysis of tacrolimus and creatinine from a single dried blood spot using liquid chromatography tandem mass spectrometry. J Chromatography B 2013;926(Pt 1):54-61.

13. Hoogtanders K, van der Heijden J, Christiaans M, Edelbroek P, van Hooff JP, Stolk LM. Therapeutic drug monitoring of tacrolimus with the dried blood spot method. J Pharm Biomed Anal 2007;44:658-64.

14. Hinchliffe E, Adaway J, Fildes J, Rowan A, Keevil BG. Therapeutic drug monitoring of ciclosporin $A$ and tacrolimus in heart lung transplant patients using dried blood spots. Ann Clin Biochem 2014;51(Pt 1):106-9.

15. Dickerson JA, Sinkey M, Jacot K, Stack J, Sadilkova K, Law YM, et al. Tacrolimus and sirolimus in capillary dried blood spots allows for remote monitoring. Pediatr Transplant 2015;19:101-6.
16. Al-Uzri AA, Freeman KA, Wade J, Clark K, Bleyle LA, Munar M, et al. Longitudinal study on the use of dried blood spots for home monitoring in children after kidney transplantation. LID 10.1111/petr.12983 doi]. Pediatric transplantation JID - 9802574 OTO - NOTNLM 0621.

17. Kloosterboer SM, de Winter BC, Bahmany S, Al-Hassany L, Dekker A, Dieleman GC, et al. Dried blood spot analysis for therapeutic drug monitoring of antipsychotics: drawbacks of its clinical application. Ther Drug Monit 2018;40:344-50.

18. Enderle Y, Foerster K, Burhenne J. Clinical feasibility of dried blood spots: analytics, validation, and applications. J Pharm Biomed Anal 2016;130:231-43.

19. Willemsen A, Knapen L, de Beer Y, Brüggemann R, Croes S, van Herpen $C$, et al. Clinical validation study of dried blood spot for determining everolimus concentration in patients with cancer. Eur J Clin Pharmacol 2018;74:465-71.

20. CLSI. Measurement Procedure Comparison and Bias Estimation Using Patient Samples; Approved Guideline - Third Edition. CLSI document EP09-A3. Wayne, PA: Clinical and Laboratory Standards Institute, 2013.

21. Abdi A, Emming G, Koster R. University Medical Centre Groningen - DBS Instruction. 2017; Available at: www.driedbloodspot. umcg.nl. Accessed December 19th, 2017.

22. CLSI. Blood collection on Filter Paper for Newborn Screening Programs; Approved Standard - Sixth Edtition. CLSI Document NBS01-A6. Wayne, PA: Clinical and Laboratory Standards Institute 2013;NBS01-A6.

23. Koster RA, Veenhof H, Botma R, Hoekstra AT, Berger SP, Bakker SJ, et al. Dried blood spot validation of five immunosuppressants, without hematocrit correction, on two LC-MS/MS systems. Bioanalysis 2017;9:553-63.

24. Koster RA, Alffenaar J-W, Greijdanus B, Uges DR. Fast LC-MS/MS analysis of tacrolimus, sirolimus, everolimus and cyclosporin $A$ in dried blood spots and the influence of the hematocrit and immunosuppressant concentration on recovery. Talanta 2013;115:47-54.

25. Koster RA. The influence of the dried blood spot drying time on the recoveries of six immunosuppressants. J App Bioanal 2015;1:395.

26. World Health Organization. Participant Manual Module 14 Blood Collection and Handling - Dried Blood Spot (DBS). 2005; Module 14: EQA(December).

27. Koster RA, Dijkers EC, Uges DR. Robust, high-throughput LC-MS/ MS method for therapeutic drug monitoring of cyclosporine, tacrolimus, everolimus, and sirolimus in whole blood. Ther Drug Monit 2009;31:116-25.

28. Bland J, Altman D. Statistical methods for assessing agreement between two methods of clinical measurement. Lancet 1986;327:307-10.

29. Passing H, Bablok W. A new biometrical procedure for testing the equality of measurements from two different analytical methods. Application of linear regression procedures for method comparison studies in Clinical Chemistry, Part I. Clin Chem Lab Med 1983;21:709-20.

30. European Medicines Agency, London, UK. Guideline on bioanalytical method validation. 2011.

31. Food and Drug Administration, US Department of Health and Human Services, Rockville, MD, USA. Guidance for Industry, Bioanalytical Method Validation. 2018.

32. Everolimus (Certican) $0.25 \mathrm{mg}$ Tablets - Summary of Product Charecteristics Update 2018, July 18th. Available at http://www. ema.europa.eu. Accessed: October 2nd, 2018. 
33. Sirolimus (Rapamune) $0.50 \mathrm{mg}$ Tablets - Summary of Product Charecteristics Update 2018, August 8th. Available at http:// www.ema.europa.eu. Accessed: October 2nd, 2018.

34. Sheiner LB, Beal SL. Some suggestions for measuring predictive performance. J Pharmacokinet Biopharm 1981;9:503-12.

35. Knapen LM, de Beer Y, Brüggemann RJ, Stolk LM, de Vries F, Tjan-Heijnen VC, et al. Development and validation of an analytical method using UPLC-MS/MS to quantify everolimus in dried blood spots in the oncology setting. J Pharm Biomed Anal 2018;149:106-13.

36. Linnet K. Evaluation of regression procedures for methods comparison studies. Clin Chem 1993;39:424-32.

37. Sadilkova K, Busby B, Dickerson JA, Rutledge JC, Jack RM. Clinical validation and implementation of a multiplexed immuno- suppressant assay in dried blood spots by LC-MS/MS. Clinica Chimica Acta 2013;421:152-6.

38. Ravaud A, Urva SR, Grosch K, Cheung WK, Anak O, Sellami DB. Relationship between everolimus exposure and safety and efficacy: meta-analysis of clinical trials in oncology. Eur J Cancer 2014;50:486-95.

39. Willemsen AE, de Geus-Oei L, De Boer M, Tol J, Kamm Y, De Jong PC, et al. Everolimus exposure and early metabolic response as predictors of treatment outcomes in breast cancer patients treated with everolimus and exemestane. Targeted Oncol 2018;13:641-8.

40. Panchal T, Spooner N, Barfield M. Ensuring the collection of high-quality dried blood spot samples across multisite clinical studies. Bioanalysis 2017;9:209-13. 